\title{
Educação em saúde: programa e canal de comunicação via WhatsApp da unidade básica de saúde do N6 para comunidade rural do sertão pernambucano
}

\author{
Aristóteles Homero dos Santos Cardona Júnior, Cleusa Wanderley de Queiroz Andrade, \\ Luciana Nogueira Mendes Caldas
}

\section{RESUMO}

Este artigo relata a experiência da divulgação de um programa de educação em saúde via transmissão de arquivos de áudio e da criação de um canal de comunicação com a comunidade local através do WhatsApp implantada na área rural de Petrolina/PE pela equipe da Unidade Básica de Saúde do Projeto de Irrigação Senador Nilo Coelho N6. 0 programa de educação em saúde intitulado "Rádio Zap do Postinho do N6" divulgado semanalmente surgiu como uma forma da equipe da Estratégia de Saúde da Família fortalecer o vínculo com a comunidade local e compartilhar o conhecimento em saúde como estratégia de enfrentamento a COVID-19. Junto com os episódios foi lançado o "WhatsApp do Postinho do N6" como um espaço de interação à distância com os usuários. A rede social visa também sanar dúvidas sobre o funcionamento da unidade, temas abordados no programa e a manutenção do cuidado de forma integral.

Palavras-chave: Comunicação; Infecções por Coronavirus; Educação em Saúde; Estratégia Saúde da Família.

\section{ABSTRACT}

This article reports the experience of broadcasting a health education program through audio files and the creation of a communication channel with the local community through WhatsApp implemented in the rural area of Petrolina / PE by the team of the Primary Health Unit from the district Projeto de Irrigação Senador Nilo Coelho N6. The health education program entitled "Rádio Zap do Postinho do N6" released weekly emerged as a way for the Family Health Strategy team to strengthen the bond with the local community and share health knowledge as a fighting strategy for COVID-19. Along with the episodes, it was launched the "WhatsApp do Postinho do N6" a space for remote interaction with users. The social network also aims to answer questions about the operation of the Health Unit, topics addressed in the program, and maintenance of comprehensive care.

Keywords: Communication; Coronavirus Infections; Health Education; Family Health Strategy.

Submissão recebida em 30 de maio de 2020

Aceito para publicação em 08 de junho de 2020.

Avaliado pelo sistema Double Blind Review com participação dos editores.
Revista da Rede APS 2020

Publicada em: 09/06/2020

DOI:10.14295/aps.v2i2.92

Aristóteles Homero dos Santos Cardona Júnior

(Universidade Federal do Vale do São Francisco, Petrolina, PE, Brasil)

Cleusa Wanderley de Queiroz Andrade

(Universidade Federal do Vale do São Francisco, Petrolina, PE, Brasil)

Luciana Nogueira Mendes Caldas

(SMS/Petrolina, Petrolina, PE, Brasil)

Correspondência para:

Aristóteles Homero dos Santos

Cardona Júnior

ari.cardona@gmail.com

Cleusa Wanderley de Queiroz Andrade

cleusawqandrade@gmail.com

Luciana Nogueira Mendes Caldas

luciananmcaldas@gmail.com 


\section{INTRODUÇÃO}

O número de celulares no Brasil ultrapassou a quantidade de habitantes. Segundo dados recentes do IBGE, o país possui 220 milhões de celulares ativos, enquanto há 207,6 milhões de habitantes. Dentro dessa realidade, a rede social WhatsApp é a mais popular, alcançando um nível recorde de presença em 99\% dos smartphones do país (Panorama Mobile Time/Opinion Box - Mensageria no Brasil Janeiro de 2020). Dessa forma, o potencial de comunicação das Redes Sociais tem sido aproveitado também como instrumento de educação. O uso de mídias sociais para disseminar informações sobre saúde tem aumentado em todo o mundo e é importante o acompanhamento dessa tendência pelos profissionais de saúde (SCHROEDER, 2017). A revolução digital tem permitido uma maior interação e assistência médica com os pacientes e a comunidade (KRYNSKI; GOLDFARB; MAGLIO, 2018).

A Tecnologia da Informação e Comunicação (TIC) facilita a comunicação, permite maior alcance entre usuários de ferramentas tecnológicas e seu possibilita a dinamização no processo de aprendizagem, construindo um espaço de reflexão (TORRES et al., 2018). A TIC constitui uma importante estratégia de pulverização do conhecimento com qualidade e permite a formação de um canal de comunicação horizontal entre os profissionais de saúde e a população, sobretudo em um cenário de distanciamento social, tendo em vista a situação pandêmica provocada pela COVID-19.

A Atenção Primária à Saúde tem como objetivo um conjunto de ações e práticas sanitárias participativas e democráticas realizadas através do trabalho em equipe para a população de uma área de abrangência. Criada nos anos 90, a Estratégia de Saúde da Família (ESF) tem como finalidade a promoção à saúde, prevenção de doenças e manutenção do cuidado de forma integral e contínua (GIOVANELLA et al., 2012). É um dos objetivos da ESF a educação em saúde de forma integrativa e emancipadora.

A Unidade Básica de Saúde N6 (UBS N6), localizada na área rural do município de Petrolina, Pernambuco, conta com uma equipe da ESF e atende a uma população de 3.905 pessoas. Sua área de abrangência tem um raio de aproximadamente 18 quilômetros e conta com apenas 03 agentes comunitárias de saúde ativas. Nesse cenário, surgiu a necessidade do compartilhamento do conhecimento de forma dialógica, criativa e participativa. A iniciativa da criação do programa educacional via WhatsApp "Rádio Zap do Postinho do N6" e do canal de comunicação "WhatsApp do Postinho do N6" pretende constituir-se estratégia de educação democrática em saúde.

Este relato de experiência tem como propósito destacar a importância do uso das redes sociais como ferramenta de educação em saúde, assim como a comunicação por meio dela como forma de fortalecimento de vínculo com o usuário do serviço de saúde, sobretudo diante do cenário de distanciamento social provocado pela COVID19. Diante do exposto, o objetivo do trabalho é descrever a experiência educativa do "WhatsApp do Postinho do N6" e "Rádio Zap do Postinho do N6" na área rural de Petrolina/PE.

\section{DeSENVOLVIMENTO}

O programa "Rádio Zap do Postinho do N6" é lançado semanalmente desde 15 de maio de 2020 e compartilhado via WhatsApp por uma lista de transmissão para os usuários da UBS N6. Além disso, há o compartilhamento do programa pelos funcionários do serviço de saúde, sendo quase que exclusivamente residentes do bairro, com os moradores da região, possibilitando maior alcance da iniciativa educacional. O programa possibilita a divulgação de atividades, aborda o conhecimento técnico/científico valorizando o conhecimento popular, contribui para a conscientização em saúde da população a que se 
dirige e estimula o compartilhamento e propagação de informações.

Cada episódio é montado com gravações individualizadas dos profissionais da equipe da UBS. O programa possui em média 10 minutos e é dividido em blocos: avisos e informações, educação em saúde através de uma linguagem simples e acessível, dúvidas dos usuários e música de artista local. Os assuntos abordados levam em conta o aspecto epidemiológico e as preocupações atuais. Diante disso, os temas discutidos foram a COVID-19, a importância do uso de máscaras e da lavagem das mãos, higienização dentária, alimentação saudável e dengue.

$\mathrm{Na}$ mesma data foi lançado também o "WhatsApp do Postinho do N6" como um canal de comunicação com a população. O uso dessa rede social tem sido cada vez mais presente entre os profissionais de saúde e seus pacientes, possibilitando melhora na comunicação, respostas imediatas e economia de tempo, buscando uma melhora do desempenho profissional (JOHNSTON et al., 2015; BENEDICTIS et al., 2019). A conta comercial informa sobre o funcionamento da unidade e permite uma interação direta com os profissionais de saúde. As mensagens que possuem o cuidado e a saúde como conteúdo central são selecionadas pela recepção e posteriormente repassadas para a equipe de saúde para a efetivação do diálogo.

O conteúdo das mensagens foi contabilizado e inserido no programa Microsoft Excel, versão 2019. O público que predomina na interação com os profissionais de saúde por meio do canal de comunicação é o feminino, $88 \%$, enquanto o masculino representa $12 \%$ das interações. A Figura 1 demonstra os principais temas abordados por porcentagem: $61 \%$ foram questionamentos sobre o funcionamento da unidade, $25 \%$ dúvidas clínicas, $13 \%$ agradecimentos e $1 \%$ sugestões.
Figura 1 | Percentual de interação dos usuários com a equipe de saúde por tema, Petrolina, PE, 2020.

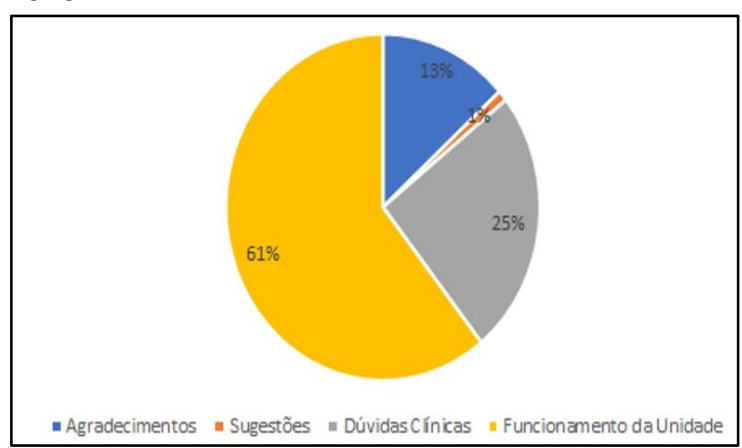

Fonte: dados levantados pelos autores.

\section{CONSIDERAÇÕES FINAIS}

O uso de estratégias educativas legítimas na realidade populacional e pautadas em uma relação horizontal, priorizando o conhecimento popular, é essencial para a construção do saber (GAZZINELLI et al., 2013). O programa "Rádio Zap do Postinho do N6" e o canal de comunicação da unidade de saúde via WhatsApp demonstram que existem diversas formas de fazer educação em saúde e que as redes sociais devem ser utilizadas como estratégia de aproximação do usuário com o serviço de saúde, assim como permitir a propagação de um saber em saúde reflexivo e emancipador. $O$ projeto tem possibilitado o fortalecimento do vínculo e o aprofundamento do conhecimento popular em saúde em um contexto de distanciamento social provocado pela pandemia da COVID-19.

Aprovada em abril de 2020, a lei 13.989 regulamentou o uso da telemedicina durante a crise causada pelo coronavírus, permitindo que casos que exigem acompanhamento contínuo sejam monitorados e que os usuários possam realizar consultas à distância, evitando a ida do paciente a um pronto-socorro, UBS ou unidade hospitalar onde haja risco de contaminação (BRASIL, 2020). 
A utilização do WhatsApp permite uma boa capilaridade na comunidade, possibilita a construção de um conhecimento e a interação e aproximação entre os profissionais de saúde e a população local. Essa rede social permite uma participação dinâmica da população, incentiva o cuidado em saúde e surge como uma alternativa de intervir no processo saúde-doença. Além disso, esse canal de comunicação da ESF permite suporte às famílias da comunidade ao possibilitar o compartilhamento de suas histórias e dúvidas sobre a saúde com os profissionais.

É importante destacar, ainda, o efeito equalizador da iniciativa em termos de diferenças sociais e econômicas ao utilizar uma ferramenta massivamente disseminada, o WhatsApp, para propagar a educação em saúde, contribuindo para diminuição das desigualdades no acesso à informação (DURMAZ et al., 2018). 


\section{NOTAS E REFERÊNCIAS}

BENEDICTIS, A. D. et al. WhatsApp in hospital? An empirical investigation of individual and organizational determinants to use. PLOS ONE, v. 14, n. 1, p. e0209873, 11 jan. 2019.

BRASIL. Lei № 13.989, de 15 de abril de 2020. Dispõe sobre o uso da telemedicina durante a crise causada pelo coronavírus (SARS-CoV-2). Diário Oficial da União. 16/04/2020. Edição: 73. Seção: 1. Página: 1. Disponível em: <http://www.in.gov.br/en/web/dou/-/lei-n-13.989-de-15-de-abril-de-2020-252726328>. Acesso em: 26 maio 2020.

DURMAZ, S. et al. A randomized controlled study with WhatsApp embedded in smoking cessation service. European Journal of Public Health, v. 28, n. suppl_4, 1 nov. 2018.

GAZZINELLI, M. F. et al. “Alô, Doutor!”: estudo-piloto de intervenção radiofônica de Educação em Saúde desenvolvida em uma área rural de Minas Gerais. Physis: Revista de Saúde Coletiva, v. 23, n. 3, p. 965985, set. 2013.

GIOVANELLA L, ESCOREL S, LOBATO LVC, NORONHA JC, CARVALHO Al. Políticas e Sistema de Saúde no Brasil. 2a ed. Rio de Janeiro: Fiocruz; 2012.

JOHNSTON, M. J. et al. Smartphones let surgeons know WhatsApp: an analysis of communication in emergency surgical teams. American Journal of Surgery, v. 209, n. 1, p. 45-51, jan. 2015.

KRYNSKI, L.; GOLDFARB, G.; MAGLIO, I. Technology-mediated communication with patients: WhatsApp Messenger, e-mail, patient portals. A challenge for pediatricians in the digital era. Archivos Argentinos De Pediatria, v. 116, n. 4, p. e554-e559, 012018.

Panorama Mobile Time/Opinion Box - Mensageria no Brasil - Janeiro de 2020. Disponível em: <https://panoramamobiletime.com.br/pesquisa-mensageria-no-brasil-fevereiro-de-2020/>. Acesso em 24 maio. 2020.

SCHROEDER, W. K. Leveraging Social Media in \#FamilyNursing Practice. Journal of Family Nursing, v. 23, n. 1 , p. 55-72, 1 fev. 2017.

TORRES, R. A. M. et al. Dialogando com os jovens sobre a obesidade através de uma Web-Rádio. $\mathbf{R}$. Saúd. Digi. Tec. Edu., Fortaleza, v. 3, n. 4, p. 30-43, jan./jun. 2018. Disponível em:<http://www.repositorio.ufc.br/handle/riufc/34325>. Acesso em 22 maio. 2020. 